\title{
Narratives are Persuasive Because They are Easier to Understand: Examining Processing Fluency as a Mechanism of Narrative Persuasion
}

\author{
Olivia M. Bullock ${ }^{1 *}$, Hillary C. Shulman ${ }^{1}$ and Richard Huskey ${ }^{2}$ \\ ${ }^{1}$ School of Communication, The Ohio State University, Columbus, OH, United States, ${ }^{2}$ Department of Communication, Center for \\ Mind and Brain, University of California, Davis, Davis, CA, United States
}

Theory suggests that people are more persuaded by information presented within a narrative. We argue there is room for greater understanding about why this may be the case. Accordingly, we 1) examine whether narratives are indeed more persuasive than non-narratives and 2) evaluate two theoretical mechanisms that could be responsible for these effects. Results from a laboratory-based, preregistered experiment $(N=554)$ support our primary argument that narratives are processed more fluently (easily) than

OPEN ACCESS

Edited by:

Satveer Kaur-Gill,

National University of Singapore,

Singapore

Reviewed by:

Pooja Jayan,

Massey University, New Zealand

Asha Rathina Pandi,

Massey University, New Zealand

${ }^{*}$ Correspondence:

Olivia M. Bullock

bullock.181@osu.edu

Specialty section:

This article was submitted to

Health Communication,

a section of the journal

Frontiers in Communication

Received: 02 June 2021

Accepted: 27 August 2021

Published: 08 September 2021

Citation:

Bullock OM, Shulman HC and Huskey $R$ (2021) Narratives are

Persuasive Because They are Easier to Understand: Examining Processing

Fluency as a Mechanism of

Narrative Persuasion.

Front. Commun. 6:719615.

doi: $10.3389 /$ fcomm.2021.719615 non-narratives, and when processing is eased, persuasion becomes more likely. This work offers a parsimonious and powerful explanation for the advantages of providing persuasive information within a narrative format and advances theory in narrative persuasion.

Keywords: narrative persuasion, processing fluency, identification, strategic communication, social cognition

\section{INTRODUCTION}

Narratives are a fixture of human communication (Fisher, 1985), and a large body of scholarship has arisen around the study of narratives and their effects on audiences (e.g., Green and Brock, 2000; Slater and Rouner, 2002; Moyer-Gusé, 2008). One area of this research focuses on narrative persuasion, the idea that narratives can impact an individual's attitudes, beliefs, and behaviors (Moyer-Gusé, 2008). Research in this area has found that exposure to narratives leads to attitude change (de Graaf et al., 2012), increased risk perceptions about health topics (Moyer-Gusé and Nabi, 2010), and prosocial behavioral intentions (McQueen et al., 2011), among other desired outcomes.

Numerous mechanisms have been proposed to explain how narratives persuade (for a review, see Moyer-Gusé, 2008). Nevertheless, our understanding of when and why narratives are persuasive remains incomplete, particularly about how persuasive appeals are more effective in narrative compared to non-narrative messages (Braddock and Dillard, 2016). Accordingly, narrative persuasion scholars have called for further exploration of the processes that facilitate narrative persuasion (de Graaf et al., 2012; Shen et al., 2015; Braddock and Dillard, 2016).

Our study answers these calls by elucidating processing fluency as a theoretical mechanism that offers a parsimonious and powerful explanation for why narratives are persuasive. Processing fluency refers to subjective feelings of ease or difficulty that occur while processing new information (Schwarz, 2010a). We examine whether narratives are indeed more persuasive than non-narratives and evaluate processing fluency as a potential mechanism that could be responsible for these effects. Importantly, we also consider processing fluency in the context of identification, another mechanism theorized to aid in narrative persuasion. Identification is defined as a process through which individuals adopt the perspective of a character and see the events of a narrative through the 
character's eyes (Cohen, 2001; Moyer-Gusé, 2008; de Graaf et al., 2012). By considering these two mechanisms in tandem, we are able to test whether processing fluency explains unique variance in persuasive message processing compared to the established mechanism of identification. In doing so, we advance theory and understanding about why, and how, narrative persuasion can be successful.

In this manuscript, we first review the literature on the persuasive power of narratives compared to non-narratives. We then discuss identification, a mechanism theorized to contribute to narrative persuasion. Next, we introduce the concept of processing fluency and articulate a theoretical argument for why processing fluency can help in understanding narrative persuasion. We report the results of a preregistered laboratory experiment that evaluates identification and processing fluency as mechanisms of narrative persuasion. We find that both explain variance in persuasive outcomes. Consistent with our preregistered hypotheses, we find that processing fluency offers a strong mechanistic explanation for persuasive outcomes. We discuss the theoretical implications of these findings in terms of well-established evaluation criteria (de Graaf et al., 2012; DeAndrea and HolbertSlater, 2017) and offer a path forward for scholars and practitioners of narrative persuasion.

\section{Narratives and Narrative Persuasion}

Research on narratives and narrative persuasion is interested in the ability of stories to educate and persuade. Narratives can be defined as "any cohesive or coherent story with an identifiable beginning, middle, and end, that provides information about scene, characters, and conflict; raises unanswered questions or unresolved conflict; and provides resolution" (Hinyard and Kreuter, 2007, p. 778). Narratives are often compared to their alternative, non-narratives, which present information in statistical or didactic formats and use reason and evidence rather than stories and exemplars to support claims (Murphy et al., 2013). Whereas narratives tell a story and contain a plot, characters, and setting, non-narratives present fact-based information outside of a story format. Common examples of non-narratives include fact sheets (e.g., Niederdeppe et al., 2011) or news articles (e.g., Oliver et al., 2012), although the vast number of forms that non-narratives can take makes the category underspecified. Scholars began to make distinctions between narrative and non-narrative messages based on experimental work suggesting that individuals process the two formats differently (Zwaan, 1994). Since that time, a significant body of research has worked to compare the relative persuasiveness of narrative messages to non-narrative messages, particularly in health contexts where non-narrative information sharing is prevalent (e.g., Murphy et al., 2013; Moyer-Gusé and Dale, 2017).

Most of the research on narratives is based on the notion that the narrative format has unique advantages over non-narrative formats. For example, because of narratives' organization, information is transferred in a way that makes the content more structured and imaginable, making narratives useful devices for organizing events, illustrating relationships, and providing examples (Glaser et al., 2009). Scholars have noted that narratives have been used in human communication for thousands of years, with some suggesting that narratives serve as educational tools for individuals to share lessons with one another (Fisher, 1985). Together, these ideas have led to suggestions that people may process narratives differently (Slater and Rouner, 2002; Moyer-Gusé, 2008), and that narratives may be easier to process than other types of information (Green and Donahue, 2018; Lee and Shin, 2019).

Making information easier to process, as narratives are hypothesized to do, should facilitate persuasion. Accordingly, research has explored the impact that narratives can have on the attitudes, beliefs, and behaviors of people who are exposed to them, a phenomenon called narrative persuasion (Moyer-Gusé, 2008). This work finds that narratives can indeed facilitate persuasion (e.g., Moyer-Gusé and Nabi, 2010; McQueen et al., 2011; de Graaf et al., 2012). However, understanding if persuasive effects are due to unique features of narratives requires a comparison to non-narratives. Studies that compare narratives to non-narratives have yielded mixed results, with some finding that narratives are more persuasive (e.g., Murphy et al., 2013; Sun et al., 2019) while others have found no differences between narratives and non-narratives on persuasive outcomes (Wang and Shen, 2019; Wirtz and Kulpavaropas, 2014). Meta-analytic research demonstrates that when studies do compare narratives to non-narratives, narratives have stronger short-term (Shen et al., 2015) and long-term persuasive impact (Oschatz and Marker, 2020). Importantly, studies that compare narratives to non-narrative messages are somewhat uncommon ${ }^{1}$, despite repeated arguments that such a comparison is vital for understanding when and why narratives, as a message format, are persuasive (e.g., Murphy et al., 2013).

Our study includes this comparison as a central feature of our design. Our goals are to 1) examine whether narratives are more persuasive than non-narratives and 2) evaluate two theoretical mechanisms that could be responsible for these effects (identification and processing fluency). Because processing a narrative is different than processing a non-narrative (Zwaan, 1994; Lee and Shin, 2019) in ways that should optimize persuasion (Green and Brock, 2000), we expect to obtain greater persuasive outcomes in the narrative format condition.

To test this expectation, we adapted a previously used risk message from Chen et al. (2016). Alterations made to this message to generate our narrative and non-narrative conditions are described in the Method section. We aimed to replicate Chen et al. (2016) similarity manipulation of identification and their measures of key mediating and outcome variables. As a result, we used the same set of persuasive outcomes from the earlier study, which also align

\footnotetext{
${ }^{1}$ We note that there are many potential reasons why narratives are not often compared to non-narratives in the literature. One issue is that the two messages must be informationally equivalent. Other potential concerns such as differences in argument quality, argument strength, number of persuasive arguments, and so on also present unique challenges when comparing persuasive narratives to a nonnarrative persuasive message.
} 
with several scales commonly used in the persuasion, health, and risk literatures. Guided by Chen et al., we expect increased perceptions of susceptibility and severity following exposure to the narrative risk message (compared to the non-narrative message), as well as increased perceived persuasiveness. Additionally, we also examine outcomes (self-efficacy and perceived knowledge) that are associated with the two literatures we integrate (narrative persuasion, processing fluency), expecting these to also be higher in the narrative conditions.

$\mathrm{H} 1$ : Those exposed to a narrative message format will report higher values on the following scales: a) susceptibility, b) severity, c) perceived persuasiveness, d) self-efficacy, and e) perceived knowledge, compared to those exposed to a non-narrative format or a no message control.

\section{Mechanisms of Narrative Persuasion}

To explain narratives' persuasive power, previous research has explored the role that the presence of characters plays in facilitating persuasion. Unlike non-narratives, which present information in abstract or aggregate terms, narratives require the use of characters to advance their plot (Hinyard and Kreuter, 2007). Further, the presence of characters can affect message processing through identification (Cohen, 2001). Identification is a process through which individuals adopt the perspective of a character and see the events of a narrative through the character's eyes (Cohen, 2001; Moyer-Gusé, 2008; de Graaf et al., 2012). When an audience member identifies with a character, he or she loses self-awareness and becomes fully merged with the feelings, perspective, motivation, and experiences of this character (Cohen, 2001). When identification occurs, persuasion becomes more likely. The theoretical basis for this relationship is derived from social cognitive theory (SCT; Bandura, 1986) and an extension (E-ELM; Slater and Rouner, 2002) of the elaboration likelihood model (ELM; Petty and Cacioppo, 1986). These theories posit that people learn through observing and imitating attractive or similar models (SCT), and that audiences that identify with a character are less likely to counterargue (E-ELM). Empirical evidence has shown that identification is linked to a variety of persuasive outcomes, including self-efficacy (Moyer-Gusé, 2008), attitude change (de Graaf et al., 2012), and behavioral intentions (Moyer-Gusé and Nabi, 2010).

Previous studies have established several ways to facilitate identification with a character. These include portraying a character in a positive light or the use of first-person perspective. Each of these manipulations is hypothesized to affect identification by increasing an audience member's ability to see events through the character (Tal-Or and Cohen, 2010). One method frequently used to alter identification is similarity between an audience member and a character (Tukachinsky, 2014; Hoeken et al., 2016).

Two propositions arise from advancing the mechanism of identification to explain narrative persuasion. The first is that, theoretically, it should not be possible to experience identification in a non-narrative persuasive message as such a message lacks a character for audiences to identify with. Accordingly, selfreported identification should be much lower after exposure to a non-narrative message relative to a narrative message. And, if identification facilitates persuasion and is maximized in narratives, non-narratives should be less persuasive. Second, within the narrative format, stories that include similar characters should be yet more successful at evoking identification than narratives with dissimilar characters (Hoeken et al., 2016).

To examine whether identification is one of the mechanisms to explain persuasive effects, we include similarity, which should increase identification, in both our narrative and non-narrative conditions. In the narrative conditions, we manipulate similarity through demographic similarity with the characters. Although we do not expect identification to occur in the non-narrative conditions, it is important to maintain informational equivalency between experimental conditions. Accordingly, we manipulate similarity through the referent group specified in both the narrative and non-narrative risk messages. In both conditions, participants answered self-report scales about identification with the referent group or character. Given that identification is considered one key mechanism of narrative persuasion (de Graaf et al., 2012), we expect that identification will mediate the relationship between the narrative format and persuasive outcomes (see Figure 1A).

$\mathrm{H} 2$ : Identification will mediate the relationship between narrative message format and a) susceptibility, b) severity, c) perceived persuasiveness, d) self-efficacy, and e) perceived knowledge, such that a positive indirect effect will be observed.

\section{The Role of Processing Fluency}

Here, we join a growing number of narrative scholars (Vaughn et al., 2010; Cohen et al., 2018; Walter et al., 2020) in arguing that narrative persuasion might be explainable via a psychological mechanism called processing fluency (for reviews, see Schwarz, 2010b; Schwarz, 2015). Briefly, processing fluency refers to subjective feelings of ease or difficulty that occur while processing new information (Schwarz, 2010a). These feelings of ease or difficulty impact individuals' perceptions of information. Specifically, when information feels like it is processed fluently, or easily, the information comes to mind quickly and is accompanied by a positive affective experience. On the other hand, when information is processed disfluently, the information requires slower, more effortful, and difficult processing. This difficulty is associated with a negative affective response (Petty et al., 2007; Schwarz, 2010b; Schwarz, 2015).

Previous research in narrative cognition, or how individuals process stories, suggests that narratives may be easier to process, and, we argue here by extension, therefore increase processing fluency. Prior research has established that narratives are easier to read and remember than non-narratives (Graesser et al., 1980), and that information contained in narratives is evaluated more favorably than information in non-narratives (Adaval and Wyer, 

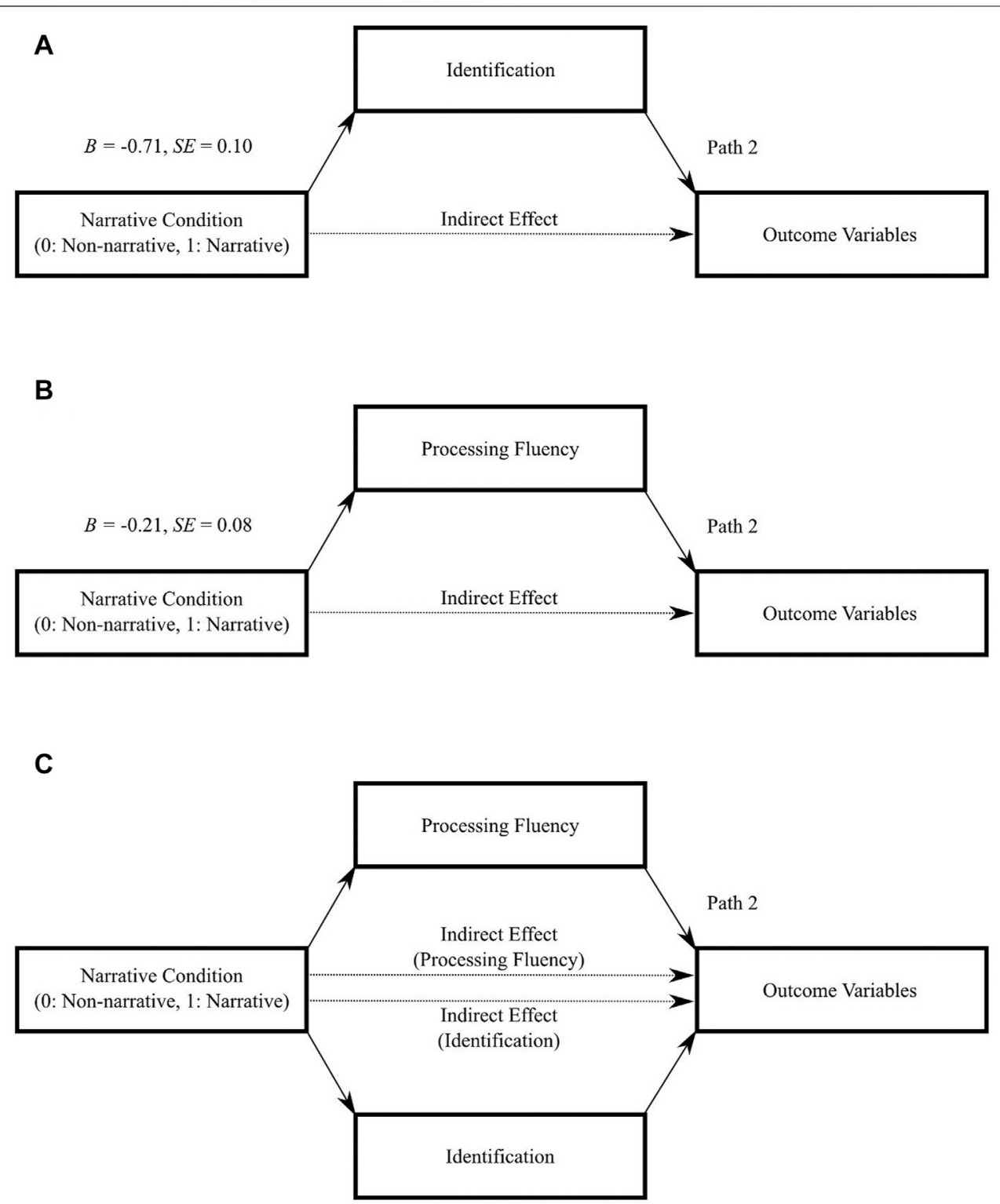

FIGURE 1 | (A) The empirical model associated with the test of hypothesis two and the path estimates provided in Table 1. This model was estimated using Model 4 (95\% bias-corrected bootstrap Cls based on 10,000 resamples) from Hayes (2013) PROCESS. (B) The empirical model associated with the test of hypothesis three and the path estimates provided in Table 1. This model was estimated using Model 4 (95\% bias-corrected bootstrap Cls based on 10,000 resamples) from Hayes (2013) PROCESS (C) A test of hypothesis four using Model 4 (Hayes, 2013, 95\% bias-corrected bootstrap Cls based on 10,000 resamples) with the "contrast = 1" option toggled on. Estimates are provided in Table 2.

1998). Because processing fluency is also associated with subjective feelings of ease and positive affective responses (Petty et al., 2007), it is possible that these effects could be explainable through fluency. Although some previous work has connected processing fluency to narrative persuasion (Vaughn et al., 2010; Cohen et al., 2018), only one study has formally tested processing fluency as a mechanism of narratives' persuasive effects. Walter et al. (2020) demonstrate that processing fluency can influence narrative persuasion by either overcoming topic ambivalence or reducing certainty during motivated processing.
Further, there are several significant theoretical, mechanistic, and outcome overlaps between narrative persuasion and processing fluency that suggest that processing fluency could contribute to narrative persuasion. For instance, both exposure to narratives and processing fluency have been associated with heuristic processing (Slater and Rouner, 2002; Alter and Oppenheimer, 2006). In the case of narrative persuasion, it has been argued that involvement with the narrative and its characters prohibits counterarguing and resistance to persuasion (Moyer-Gusé, 2008). Similarly, processing fluency is also thought to operate through heuristic processing (Alter and Oppenheimer, 
2006). This is based on the notion that information that is easy to process engenders positivity and, as a result, receives less scrutiny (Schwarz, 2010b).

If narrative persuasion and processing fluency are both associated with heuristic processing, then these concepts should lead to similar outcomes. In support of this claim, when information is processed fluently, a variety of positive associations are triggered and attributed to the information itself. These associations include feelings of knowing (Schwartz and Metcalfe, 1994), safety (Song and Schwarz, 2009), confidence (Koriat, 2008), liking (Petty et al., 2007), and interest and efficacy (Shulman and Sweitzer, 2018). Similar outcomes are also associated with narrative persuasion, including reduced reactance, greater liking, more message-consistent perceptions of risk or vulnerability, and increased self-efficacy (Moyer-Gusé, 2008). These similarities offer the possibility that narrative persuasion could be operating through the mechanism of processing fluency. Specifically, narratives might be more persuasive because the message format facilitates more fluent processing compared to non-narratives.

To test this proposition, we incorporated an experimental manipulation that would produce variance in processing fluency independent of the message content within the narrative and non-narrative formats. This approach was important to avoid confounding changes in processing fluency with changes in message format. We manipulated processing fluency by overlaying white noise onto an audio message to impair processing and create a more disfluent experience when this noise was present (for an extended rationale, see Dragojevic and Giles, 2016). We argue that if processing fluency is a mechanism of narrative persuasion, then the narrative format should produce an easier processing experience irrespective of the processing fluency manipulations. And, when information is processed fluently, persuasion should be more likely (see Figure 1B).

H3: Processing fluency will mediate the relationship between narrative message format and a) susceptibility, b) severity, c) perceived persuasiveness, d) self-efficacy, and e) perceived knowledge, such that a positive indirect effect will be observed.

Finally, to offer a convincing case that establishes processing fluency as a viable mechanism of narrative persuasion, we test the predictive power of processing fluency alongside identification. Conceptually, processing fluency should be distinct from identification because processing fluency refers specifically to the ease or difficulty of processing information and does not require nor depend on the presence of characters, nor features of these characters (e.g., similarity) to occur. Moreover, if processing fluency offers a distinct explanation for why narratives are persuasive, it then becomes critical to contextualize the strength of this relationship in contrast to a well-established mediator of narrative persuasion. This contrast offers a first step towards addressing a broader theoretical question in narrative persuasion: Is it the narrative format (compared to non-narrative formats) or features of the narrative content (such as character identification) that more strongly contributes to persuasion? To begin addressing this question, we compare processing fluency with identification. If processing fluency is a distinct and stronger mechanism, then including it in future studies of narrative persuasion should increase the explanatory power of our models (see Figure 1C).

H4: Processing fluency will be a stronger mechanism of the relationship between narrative message format and a) susceptibility, b) severity, c) perceived persuasiveness, d) self-efficacy, and e) perceived knowledge, than identification.

\section{MATERIALS AND METHODS}

Answering recent calls for open science practices in communication research (Dienlin et al., 2020), our hypotheses, experimental design, sample size, stimuli, and analysis plan were preregistered (https://osf. io/rgduq/). This Open Science Framework repository includes materials, data, and syntax to reproduce our results (some components are currently hidden to ensure blind review). All study materials and procedures were approved and determined exempt by the Institutional Review Board (IRB) at The Ohio State University.

\section{Participants}

Participants were 554 undergraduate students. Students were eligible to participate in the study if signed up and attended an in-person laboratory session in exchange for course credit. A power analysis using $\mathrm{G}^{\star}$ Power (Faul et al., 2009) was used to determine sample size before beginning data collection. The power analysis was conducted with effect sizes of identification ( $d=0.37$, Tukachinsky, 2014) and processing fluency $(d=0.38$, Dragojevic and Giles, 2016) established in prior research to detect differences between groups with $85 \%$ power, 0.05 alpha, and two-tailed testing. Study participants (64.6\% female) ranged in age from 18 to $48(M=20.18$; $S D=$ 2.52 ) and reported their race as $61.7 \%$ White, $23.1 \%$ Asian, $8.8 \%$ Black, and $6.4 \%$ Other. Nine participants were excluded from data analysis because of technical difficulties, including an inability to hear the audio messages or malfunctions with the experiment.

\section{Procedure}

Participants were seated in front of a desktop computer with standardized volume and provided with standardized headphones. After providing informed consent through an online form, participants were randomly assigned to condition using Qualtrics random assignment feature in a 2 (narrative vs. non-narrative) $\times 2$ (high vs. low identification $) \times 2$ [processing fluency high (no white noise) vs. low (white noise) +1 (no message control) between-subjects experimental design. Participants were only assigned to one condition. All participants in experimental conditions listened to an audio message about the health risks of consuming too much caffeine (see below). Participants were not able to advance to the next part of the study until they listened to the entire message 
(duration: 3:40). Following message exposure, participants responded to self-report measures. In the no message control condition $(n=41)$, participants only answered self-report measures and were not exposed to a message. The study took about $40 \min (M=36.08 ; S D=25.60){ }^{2}$

\section{Stimuli}

The materials used in this study were adapted from a previously published paper authored by Chen et al. (2016), which describe a fictional character experiencing a caffeine overdose. $^{3}$ As the authors note, caffeine overdose was chosen because it poses risks for a variety of audiences and is similarly relevant to both males and females. For this study, these stimulus materials were chosen for several reasons. First, we wanted to conduct a replication of a study that had successfully manipulated identification and produced theoretically consistent findings. Given that replication is vital to a cumulative communication science (McEwan et al., 2018), this allowed us to examine the replicability of a core finding in the narrative persuasion literature. Second, we chose the Chen et al. (2016) study because they manipulated identification through similarity between a subject and a character, which has been identified as a consistently effective way to manipulate identification (Tukachinsky, 2014; Hoeken et al., 2016;).

\section{Narrative vs. Non-narrative}

The present study was interested in comparing the effects of narrative to non-narrative formats. Given that the original study did not have a non-narrative condition, we created one by removing any references to plot or characters (Hinyard and Kreuter, 2007) and only extracted fact-based information (for sample stimuli, see the preregistration document). For example, in the narrative condition ( $n=$ 332), a character becomes "drenched in sweat, burning up, and hyperventilating." To create the non-narrative version $(n=181)$, this text was rewritten to remove any references to characters: "More serious side effects are fast heartbeat, difficulty breathing, and hyperventilation." Then, the paragraphs of the non-narrative condition were numbered and randomized to remove any chronological arc. This resulted in a message like a fact sheet. Lastly, to fully cross our design and be able to measure identification in narrative and non-narrative conditions with the scale described below, referent groups were inserted in the nonnarrative format. For instance, in the high identification non-narrative condition $(n=91)$, the message referred to the risks of caffeine overdose among students. In the low identification non-narrative condition $(n=90)$, the message referred to the risks of caffeine overdose among workers. Word count, Flesch reading ease, and Flesh-Kinkaid reading grade level were held constant across all messages.

${ }^{2}$ Our standard deviation was high because of our control condition.

${ }^{3} \mathrm{We}$ thank these scholars for generously sharing their materials with us.

\section{Identification}

The materials from Chen et al. (2016) manipulated identification through gender- and age-matching, such that a person whose gender and age matched that of the character in the message experienced the greatest identification, and a person whose gender and age was mismatched experienced the least identification. This manipulation was largely maintained in the present study, with additional changes made to maximize identification. For example, in the age-matched conditions $(n=257)$, the character was named one of the most popular names from 18 years ago (Lauren/Andrew) and was described as a student at the university where this study took place. In the age mismatched conditions $(n=256)$, the character was named one of the most popular names from 50 years ago (Sandra/Robert) and was described as an older person who lived in a different state. We expected our manipulation to function such that participants who were exposed to a narrative message about a similar character would experience greater identification than participants who were exposed to a non-narrative message, or a narrative message about an older, opposite-sex character.

A manipulation check using the six-item identification scale from Chen et al. (2016) was used to assess whether this manipulation was successful. A two-way analysis of variance (ANOVA) was used to test whether age and gender matching affected identification. This analysis revealed that participants in conditions with both age, $F(1,315)=5.96, p=0.02, \eta^{2}=0.02$, and gender matching, $F(1,315)=5.30, p=0.02, \eta^{2}=0.02$, functioned in the intended ways. Specifically, college students identified more with the younger referent $(M=4.08, S D=1.12)$ than the older referent $(M=3.78, S D=1.15)$, and gender-matched conditions produced higher identification $(M=4.07, S D=1.03)$ than mismatched conditions $(M=3.79, S D=1.13)$. There was, however, no interaction effect, $F(1,315)=0.68, p=0.410, \eta^{2}=0.00$. Because age and gender matching did not interact, or in other words matching on both age and gender did not lead to significantly more identification than matching on one (or neither), we used age as the similarity factor. Given that the non-narrative condition included an age cue (student versus worker) but no gender cue, collapsing in this way allowed all format conditions to be included in analyses.

\section{Processing fluency}

The final factor manipulated was processing fluency. After generating text versions of all messages, the materials were read aloud and recorded as audio files. The non-narratives and the narratives told from the perspective of a female character were recorded by a female voice actor, and the narratives told from the perspective of a male character were recorded by a male voice actor. All recordings were the same duration (3:40) and were normalized at $70 \mathrm{~dB}$ sound pressure level. To create the low processing fluency conditions $(n=261)$, guided by Dragojevic and Giles (2016), these recordings were mixed with white noise in a +10 signal-to-noise ratio. This manipulation was chosen to induce variance in self-reports of processing fluency without needing to alter the content of the messages. The high processing fluency conditions $(n=248)$ were unaltered audio recordings. 
Consistent with findings from previous work (Dragojevic and Giles, 2016), a manipulation check using the processing fluency scale from Shulman and Sweitzer, 2018, confirmed that this manipulation functioned as intended, $F(1,507)=26.94, p<$ $0.001, \eta^{2}=0.05$. Specifically, those in the white noise conditions reported significantly lower processing fluency $(M=5.89$, $S D=1.02)$ compared to those in the no-white noise conditions $(M=6.29, S D=0.69)$.

\section{Measures}

All measures were assessed using 1-7 Likert scales, wherein higher scores indicate stronger agreement with the concept being measured. Questions assessing mediating states were asked first and the order of subsequent outcome measures was randomized.

\section{Mediating Variables}

Identification was measured with six items from Chen et al. (2016), who adapted a measure previously reported by Murphy et al., $2013(M=4.19, S D=1.09$, Cronbach's $\alpha=$ 0.81, McDonald's $\omega=0.82$ ). Questions included whether participants felt they were similar to, knew well, identified with, had a connection to, had a good understanding of, and could get inside the head of the character or referent in the message. Processing fluency was measured with three items from Shulman and Sweitzer, $2018(M=6.09, S D=0.90, \alpha=0.73, \omega=$ 0.73 ), which included questions about ease of understanding and listening to the message.

\section{Outcome Variables}

Four items measuring susceptibility $(M=2.67, S D=1.22, \alpha=0.83$, $\omega=0.82)$ and five items measuring severity $(M=6.31, S D=0.75$, $\alpha=0.92, \omega=0.92$ ) were assessed using scales from Chen et al. (2016). The susceptibility scale asked participants how likely they believed that they were to experience a caffeine overdose that would require medical attention. The severity scale asked participants how much they agreed that caffeine overdose could pose a serious threat to their health. Perceived persuasiveness was measured with a semantic differential scale used by Chen et al. (2016; $M=5.46, S D=0.98, \alpha=0.91, \omega=0.92)$. Self-efficacy $(M=5.99, S D=0.79, \alpha=0.79, \omega=0.79)$ and perceived knowledge $(M=4.93, S D=0.99, \alpha=0.75, \omega=0.73)$ were measured with scales adapted from (Shulman and Sweitzer, 2018). The self-efficacy scale included four questions about participants' beliefs that they could understand and avoid caffeine overdose. The perceived knowledge scale included five questions about participants' perceptions of being informed about caffeine overdose facts.

\section{Demographic Variables}

Participants were also asked to report demographic information, including age, gender, and race. Because the stimulus materials were about a worker who consumes too much caffeine, they also self-reported their weekly consumption of caffeinated drinks $(M=6.17, S D=5.87)$ and work experience. Forty-seven percent of respondents reported working a job outside of being a student for an average of $17.63 \mathrm{~h}$ per week $(S D=9.07)$.

\section{RESULTS}

Hypothesis one predicted that those exposed to information in a narrative format would report higher values on the scales of susceptibility, severity, perceived persuasiveness, self-efficacy, and perceived knowledge than those exposed to information in a non-narrative format or no message control. To test this hypothesis, we first conducted a one-way ANOVA to examine the effect of exposure to narratives relative to a no message control on our outcome measures. Exposure to a narrative significantly increased perceptions of severity $[F(1,366)=80.52, p<0.001$; $\left.\eta^{2}=0.18\right]$, self-efficacy $\left[F(1,366)=17.34, p<0.001 ; \eta^{2}=0.05\right]$, and perceived knowledge $\left[F(1,366)=88.14, p<0.001 ; \eta^{2}=0.19\right]$ relative to a no message control. Susceptibility was not significant. ${ }^{4}$ Then, to examine the effect of narrative versus non-narrative formats on outcome variables, we conducted an additional MANOVA $^{5}$ with narrative versus non-narrative format on outcomes. There was a statistically significant difference in persuasive outcomes based on message format, $F$ $(5,491)=2.51, p=0.04 ;, \eta^{2}=0.005$. Further analysis revealed that there was a significant difference between narrative and nonnarrative exposure for only one outcome, persuasiveness. However, this difference was in the opposite direction as predicted, such that those in the non-narrative conditions actually reported greater $\left[F(1,495)=5.21, p=0.02 ; \eta^{2}=0.01\right]$ persuasiveness $(M=5.60, S E=0.07)$ than those in the narrative conditions $(M=5.38, S E=0.05)$. There were no other significant differences observed. Thus, support for $\mathrm{H} 1$ was mixed: The narrative condition did produce more persuasive outcomes relative to the no message control, but, contrary to expectations, not compared to the non-narrative condition.

Hypothesis two predicted that identification would mediate the relationship between narrative message format and the outcomes of susceptibility, severity, perceived persuasiveness, self-efficacy, and perceived knowledge. This hypothesis was tested using Model 4 of the PROCESS macro for SPSS with 10,000 bootstrapped resamples (Hayes, 2013). The model was constructed with narrative format (narrative vs. non-narrative) as the predictor variable with non-narrative format as the referent group, identification as the mediating variable, and susceptibility, severity, perceived persuasiveness, self-efficacy, and perceived knowledge as outcome variables (see Figure 1A). Separate models were conducted for each outcome variable. With this analysis, non-zero positive indirect effects would indicate support for H2. Results for this hypothesis were mixed. This is largely because the association between the narrative format and identification was opposite from expectations $(B=-0.71, S E=$ $0.10, t=-7.44, p<0.05)$, such that those in the non-narrative condition reported higher identification than those in the

\footnotetext{
${ }^{4}$ Participants in the no message control did not respond to the perceived persuasiveness scale, as they were not exposed to a message to assess.

${ }^{5}$ Our pre-registration document states that $\mathrm{H} 1$ would be tested with a series of ANOVAs. However, given that five different tests potentially inflates family wise error rates, thereby making Type I error more likely, we opted for a more conservative MANOVA test. However, we ran the five ANOVAs and the pattern of results is identical.
} 
TABLE 1 | Results from the mediation analyses for hypotheses 2 and 3.

\begin{tabular}{|c|c|c|c|c|}
\hline \multirow[t]{2}{*}{ Outcomes } & \multirow[t]{2}{*}{ Path 2 B (SE) } & \multirow[t]{2}{*}{$R^{2}$} & Indirect Effect & \multirow{2}{*}{$\begin{array}{l}95 \% \text { CI } \\
\text { UL, LL }\end{array}$} \\
\hline & & & $B(S E)$ & \\
\hline Severity & $0.09(0.03)^{\star \star}$ & 0.02 & $-0.07(0.02)$ & $-0.12,-0.03$ \\
\hline Persuasiveness & $0.26(0.04)^{\star \star \star}$ & 0.08 & $-0.19(0.04)$ & $-0.28,-0.11$ \\
\hline Self-Efficacy & $0.02(0.03)$ & 0.004 & $-0.02(0.03)$ & $-0.07,-0.03$ \\
\hline \multicolumn{5}{|c|}{ H3: Processing fluency as a mediator } \\
\hline \multirow[t]{2}{*}{ Outcomes } & \multirow[t]{2}{*}{ Path 2 B (SE) } & \multirow[t]{2}{*}{$R^{2}$} & Indirect Effect & $95 \% \mathrm{Cl}$ \\
\hline & & & $B(S E)$ & UL, LL \\
\hline Susceptibility & $-0.17(0.06)^{\star \star}$ & 0.02 & $-0.04(0.02)$ & $-0.08,-0.005$ \\
\hline Severity & $0.17(0.03)^{\star \star \star}$ & 0.05 & $0.04(0.02)$ & $0.007,0.071$ \\
\hline
\end{tabular}

Note: Path 2 denotes the relationship between the mediator and outcomes. All models were run using Model 4 (Hayes, 2013, 95\% bias-corrected bootstrap Cls based on 10,000 resamples) where non-zero indirect effects indicate support for the model proposed.

narrative condition (Path 1). The relationship between identification and outcomes, however, was largely in the expected direction (Path 2, Table 1) such that higher identification was associated with more persuasive outcomes for all outcomes except self-efficacy. Finally, the test of indirect effects, used to test the hypothesis that identification mediates the relationship between narrative format and persuasion was consequently unsupported as well. Although there was evidence of mediation, this evidence was opposite of predictions, indicating that the non-narrative condition was more successful at inducing persuasion through identification than the narrative condition.

Hypothesis three predicted that processing fluency would mediate the relationship between the narrative conditions and susceptibility, severity, perceived persuasiveness, self-efficacy, and perceived knowledge. To test this hypothesis, we used the same models as in H2 (see Figure 1B), but used processing fluency as the mediating variable instead of identification. With this analysis, non-zero positive indirect effects would indicate support for H3. We largely found support for this mediation model in the hypothesized direction across four out of the five outcomes (Table 1). Specifically, exposure to narratives increased processing fluency $(B=0.21, S E=0.08, t=2.50, p<0.05$, Path 1$)$, which, in turn, yielded more persuasive outcomes for all proposed outcomes except for susceptibility (Path 2, Table 1). Furthermore, the indirect effects used to test mediation offered further support for $\mathrm{H} 3$ for four of the five outcomes. Thus, consistent with $\mathrm{H} 3$, the evidence supports the claim that narratives produced easier processing, and when processing was easier, more persuasive outcomes were reported.

Lastly, H4 predicted that processing fluency would be a stronger mechanism of the relationship between narrative format and persuasive outcomes. To determine if processing fluency was a stronger mechanism of narrative persuasion, we used Hayes' PROCESS Model 4 with 10,000 bootstrapped resamples, with both identification and processing fluency included as mediators and the contrast option toggled on (see Figure 1C). The contrast option in Hayes' PROCESS macro enables researchers to determine whether indirect effects are significantly different from one another (represented by a nonzero difference) and the relative strength of each effect. The results from this analysis (Table 2) indicate that processing fluency and identification produced significantly different effects for four out of the five outcomes (all but self-efficacy). Further, the directionality of these effects across all five outcomes indicate that processing fluency represents the larger, or stronger, effect. The only outcome that was not significantly different between identification and processing fluency was self-efficacy. Thus, H4 was largely supported.

\section{DISCUSSION}

The purpose of this experiment was to enhance our understanding of the mechanisms that facilitate narrative persuasion, and in doing so, advance theory in this domain. To highlight these theoretical pursuits, this discussion section is organized in accordance with Slater and Gleason (2012) and DeAndrea and Holbert (2017) evaluation criteria for theoretical contributions.

\section{Addressing Conceptual Issues}

One of the difficulties of studying the concept of narrative persuasion is that there is so much variance both within and between stories that it is difficult to pinpoint the message features responsible for producing persuasive effects. For theorists interested in explaining narrative persuasion, and practitioners interested in reproducing these persuasive effects, this lack of specificity is problematic. This was the conceptual concern that prompted this investigation. We 
TABLE 2 | Results from the contrast of parameter estimates from $\mathrm{H} 4$

\begin{tabular}{|c|c|c|c|c|c|c|}
\hline Outcomes & $\begin{array}{l}\text { Indirect effect } \\
\text { of identification } \\
\text { B (SE) }\end{array}$ & $\begin{array}{l}95 \% \text { Cl } \\
\text { UL, LL }\end{array}$ & $\begin{array}{l}\text { Indirect effect } \\
\text { of processing } \\
\text { fluency B } \\
\text { (SE) }\end{array}$ & $\begin{array}{l}95 \% \text { Cl } \\
\text { UL, LL }\end{array}$ & $\begin{array}{l}\text { Indirect effect } \\
\text { contrast B } \\
\text { (SE) }\end{array}$ & $\begin{array}{l}95 \% \mathrm{Cl} \\
\text { UL, LL }\end{array}$ \\
\hline Susceptibility & $-0.32(0.06)$ & $-0.44,-0.22$ & $-0.06(0.03)$ & $-0.11,-0.01$ & $0.26(0.06)$ & $0.14,0.40$ \\
\hline Severity & $-0.05(0.02)$ & $-0.09,-0.008$ & $0.03(0.02)$ & $0.005,0.06$ & $0.08(0.03)$ & $0.03,0.13$ \\
\hline Persuasiveness & $-0.14(0.04)$ & $-0.23,-0.07$ & 0.07 (0.03) & $0.02,0.13$ & $0.21(0.05)$ & $0.13,0.31$ \\
\hline Self-Efficacy & $0.009(0.03)$ & $-0.04,0.06$ & $0.04(0.02)$ & $0.006,0.08$ & $0.03(0.03)$ & $-0.03,0.09$ \\
\hline P. Knowledge & $-0.19(0.04)$ & $-0.28,-0.12$ & $0.04(0.02)$ & $0.007,0.09$ & $0.24(0.04)$ & $0.16,0.33$ \\
\hline
\end{tabular}

Note: The first two columns denote the indirect effect of narrative format on outcomes through identification and the associated $95 \%$ confidence interval for this parameter. The second two columns denote the indirect effect of narrative format on outcomes through processing fluency and the associated 95\% confidence interval for this parameter. The last two columns provide a contrast of parameter estimates. The estimate for the effect of identification is subtracted from the estimate of the effect for processing fluency. A positive coefficient indicates that processing fluency produced the larger effect. All models were run using Model 4 (Hayes, 2013, 95\% bias-corrected bootstrap Cls based on 10,000 resamples) with the "contrast = 1" option toggled on.

${ }^{*} p<0.05,{ }^{* *} p<0.01,{ }^{* * *} p<0.001$.

argued that to understand why narrative persuasion is successful, the effects attributable to the narrative format need to be disentangled from the effects attributable to the narrative content.

Identification was examined here because of its prominence and relative success in the narrative persuasion literature (Cohen, 2001). In this experiment, we manipulated identification in ways that were independent of the narrative format manipulation using a common manipulation from this literature-similarity (Hoeken et al., 2016). Similarity allowed us to manipulate identification in both a narrative and a nonnarrative format. This would not have been possible by using many other types of identification manipulations (for example, first- or third-person perspective), which require a narrative format. By manipulating similarity in a narrative and a nonnarrative, we were able to distinguish the persuasive effects of identification from the persuasive effects of simply using a narrative format. Contrary to $\mathrm{H} 2$, we found that participants self-reported higher levels of identification with a similar referent in the non-narrative condition compared to the similar character in the narrative condition. Unfortunately, our theoretically unexpected finding might be common in the literature. Meta-analyses (e.g., Tukachinsky, 2014) suggest that manipulations of identification work as anticipated in slightly more than half of cases. Here, we were unable to replicate the persuasive effects of identification through similarity within a narrative format even though we chose stimulus materials that have worked in the past with the same population of participants (i.e., undergraduate students, Chen et al., 2016). These meta-analytic trends, coupled with the results from our experiment, suggest that identification, as a mechanism, may be more successful when it is conceived of as a content effect rather than a format effect. In other words, similarity may induce persuasion through identification, but this process may not need to be confined within a narrative format to be successful. This conclusion is consistent with longstanding (e.g. Cantor et al., 1976) research demonstrating that messages are more persuasive when the source is perceived as similar to the target, even when that message is presented in a non-narrative format.
Similarly, another important consideration of this work is the need to disentangle similarity and identification as persuasive mechanisms. In the present study, we manipulated identification through similarity, consistent with previous work (de Graaf et al., 2012; Hoeken et al., 2016). However, other researchers have argued that identification and similarity should be thought of as distinct psychological constructs (Moyer-Gusé, 2008). Indeed, it could be said that a limitation of our study is that our measure of identification (replicated from Chen et al., 2016, who used the same measure as Murphy et al., 2013) possibly captured similarity instead of identification. If this is the case, our results show support for similarity as a mechanism of narrative persuasion rather than as a manipulation of the mechanism of identification. This conceptual ambiguity leads us to join in the calls from other scholars to further distinguish between the many proposed mechanisms of narrative persuasion (Brown, 2015; MoyerGusé, 2015). Parsing apart effects of similarity from effects of identification represents a promising future direction for this work.

\section{Elucidating Mechanism}

As a second theoretical contribution, we offered and tested the mechanism of processing fluency as a causal explanation for why narratives might produce persuasion. We further argued that, relative to identification, processing fluency should be a stronger explanatory mechanism for narrative persuasion. Identification as a causal mechanism is part of a complicated, multifaceted process: Individuals must be exposed to a message that contains characters, those characters should be likeable, relatable, empathetic, or similar to audience members to maximize identification, and through the experience of losing oneself in the narrative, persuasive effects become possible (Moyer-Gusé, 2008). This explanation for narrative persuasion is complex and relies on several moving parts to be successful. Importantly, this explanation also does not sufficiently separate the advantage of the narrative format from the advantage of certain types of content within these stories.

By comparison, processing fluency offers more parsimonious explanation of narrative persuasion. Put simply, when information feels easier to process, a variety of positive 
associations are triggered and attributed to the presented information (Schwarz, 2010a). Thus, rather than rely on the argument that narratives are effective due to the presence of specifically crafted characters or content, processing fluency offers that narratives may just be easier to process, which facilitates persuasion. Consistent with this expectation, the results related to $\mathrm{H} 3$ demonstrated that narrative formats were easier to process than non-narrative formats, and when processing was eased, more persuasive outcomes were obtained.

\section{Theory Comparison}

In addition to elucidating processing fluency as a plausible mechanism of narrative persuasion, we contextualized the strength of this relationship by comparing the outcomes of processing fluency against identification. The results from $\mathrm{H} 4$ reveal that processing fluency had greater explanatory power than identification. Moreover, when comparing identification to processing fluency as competing mechanisms of narrative persuasion, processing fluency consistently produced indirect effects in the expected direction, and these indirect effects were significantly stronger than those associated with identification. Thus, this comparison revealed that processing fluency empirically outperformed identification.

\section{Implications for Narrative Persuasion Research}

ELM-based explanations for narrative persuasion often argue that identification in narratives facilitates message elaboration and reduces counterarguing (Moyer-Gusé, 2008). One complication is that, in a narrative context, "a clean distinction between central and peripheral processes is no longer discernable" (Slater and Rouner, 2002, p.177). If central processing is critical for durable and long-lasting persuasion (Petty and Cacioppo, 1986), then it is unclear if, how, and when, narrative identification facilitates central processing. The findings of this experiment offer a potential answer for the role of central processing in narrative persuasion. Specifically, previous findings suggest that increased processing fluency leads to positive evaluations of the information at hand. In turn, positive evaluations lead to greater message elaboration (Petty and Briñol, 2008). As a result, our findings raise the possibility that greater processing fluency associated with narrative messages could, in fact, lead to more central processing. Future work should investigate this relationship in detail.

It may be that not all narratives are equally persuasive. Audience knowledge (Yeshurun et al., 2017), preexisting beliefs (Huskey et al., 2017), and a variety of other content and structural features contribute to successful narratives. These findings demonstrate that persuasive narratives depend on the interaction between message content and audience characteristics. How are message designers to know, in advance if they have successfully developed a persuasive narrative? Research shows that more persuasive health narratives better synchronize neural processing among audience members (Imhof et al., 2017). Such findings illuminate the biological basis of narrative persuasion. That said, it is rather impractical (and expensive) to scan the brains of potential audiences when designing a campaign. Our results suggest that processing fluency may serve as a low-cost alternative during message development. Good narratives should lead to higher processing fluency, which should ultimately lead to persuasion. As such, self-reported processing fluency can help campaign designers test effective messages.

\section{Limitations}

We acknowledge that there are multiple mechanisms that are theorized to underlie the effects of narrative persuasion, and identification is only one. We chose to compare processing fluency to identification because of the clear conceptual distinctions between these two constructs. However, future work should consider processing fluency alongside other mechanisms of narrative persuasion, particularly transportation (Green and Brock, 2000). Both transportation and processing fluency refer to individuals' information processing experience. Thus, disentangling these two concepts is the critical next step for future research. We also recognize the limitations of using a single message design with a student sample in this study. Future research should consider testing multiple messages among generalizable populations. We also recognize that our use of the term "non-narrative" throughout this study is necessarily underspecified due to the many types of non-narrative messages that exist. Future work should aim to delineate non-narratives more clearly as a distinct category. Finally, we acknowledge that our study explored narrative persuasion through an audio format, which has been less commonly studied compared to text-based or audiovisual narrative messages. Later research should explore how presentation mode might amplify or mitigate the relationships observed in this study. Despite these limitations, our study adds evidence to the small but growing body of literature (Vaughn et al., 2010; Cohen et al., 2018; Walter et al., 2020) investigating the role of processing fluency in narrative persuasion.

\section{DATA AVAILABILITY STATEMENT}

The datasets presented in this study can be found in online repositories. The names of the repository/repositories and accession number(s) can be found below: https://osf.io/rgduq/.

\section{ETHICS STATEMENT}

The studies involving human participants were reviewed and approved by the Ohio State University Institutional Review Board. The patients/participants provided their written informed consent to participate in this study.

\section{AUTHOR CONTRIBUTIONS}

OB and HS conceived of the original study concept. OB, HS, and $\mathrm{RH}$ developed the research design. OB oversaw data collection and analyzed the data. OB wrote the manuscript with contributions from HS and RH. 


\section{REFERENCES}

Adaval, R., and Wyerjr, R. (1998). The Role of Narratives in Consumer Information Processing. J. Consumer Psychol. 7 (3), 207-245. doi:10.1207/ s15327663jcp0703_01

Alter, A. L., and Oppenheimer, D. M. (2006). Predicting Short-Term Stock Fluctuations by Using Processing Fluency. Proc. Natl. Acad. Sci. 103 (24), 9369-9372.

Bandura, A. (1986). Social Foundations of Thought and Action: A Social Cognitive Theory. Hoboken, NJ: Prentice-Hall.

Braddock, K., and Dillard, J. P. (2016). Meta-analytic Evidence for the Persuasive Effect of Narratives on Beliefs, Attitudes, Intentions, and Behaviors. Commun. Monogr. 83 (4), 446-467. doi:10.1080/ 03637751.2015 .1128555

Brown, W. J. (2015). Examining Four Processes of Audience Involvement with Media Personae: Transportation, Parasocial Interaction, Identification, and Worship. Commun. Theor. 25 (3), 259-283. doi:10.1111/comt.12053

Cantor, J. R., Alfonso, H., and Zillmann, D. (1976). The Persuasive Effectiveness of the Peer Appeal and a Communicator's First-Hand Experience. Commun. Res. 3 (3), 293-310. doi:10.1177/009365027600300304

Chen, M., Bell, R. A., and Taylor, L. D. (2016). Narrator Point of View and Persuasion in Health Narratives: The Role of Protagonist-Reader Similarity, Identification, and Self-Referencing. J. Health Commun. 21 (8), 908-918. doi:10.1080/10810730.2016.1177147

Cohen, E. L., Wasserman, J. A., Schlue, L. M., Keely, C., and Russell, A. (2018). Seeing Is Believing: The Role of Imagery Fluency in Narrative Persuasion through a Graphic Novel. Psychol. Popular Media 9, 176-183. doi:10.1037/ ppm0000218

Cohen, J. (2001). Defining Identification: A Theoretical Look at the Identification of Audiences with Media Characters. Mass Commun. Soc. 4 (3), 245-264. doi:10.1207/S15327825MCS0403_01

de Graaf, A., Hoeken, H., Sanders, J., and Beentjes, J. W. J. (2012). Identification as a Mechanism of Narrative Persuasion. Commun. Res. 39 (6), 802-823. doi:10.1177/0093650211408594

DeAndrea, D. C., and Holbert, R. L. (2017). Increasing Clarity where it Is Needed Most: Articulating and Evaluating Theoretical Contributions. Ann. Int. Commun. Assoc. 41 (2), 168-180. doi:10.1080/23808985.2017.1304163

Dienlin, T., Johannes, N., Bowman, N. D., Masur, P. K., Engesser, S., Kümpel, A. S., et al. (2020). An Agenda for Open Science in Communication. J. Commun. 71, 1-26. jqz052. doi:10.1093/joc/jqz052

Dragojevic, M., and Giles, H. (2016). I Don't like You Because You're Hard to Understand: The Role of Processing Fluency in the Language Attitudes Process. Hum. Commun. Res. 42, 396-420. doi:10.1111/hcre.12079

Faul, F., Erdfelder, E., Buchner, A., and Lang, A.-G. (2009). Statistical Power Analyses Using $\mathrm{G}^{\star}$ Power 3.1: Tests for Correlation and Regression Analyses. Behav. Res. Methods 41 (4), 1149-1160. doi:10.3758/BRM.41.4.1149

Fisher, W. R. (1985). The Narrative Paradigm: In the Beginning. J. Commun. 35 (4), 74-89. doi:10.1111/j.1460-2466.1985.tb02974.x

Glaser, M., Garsoffky, B., and Schwan, S. (2009). Narrative-based Learning: Possible Benefits and Problems. Communications 34 (4). doi:10.1515/ COMM.2009.026

Graesser, A. C., Hoffman, N. L., and Clark, L. F. (1980). Structural Components of reading Time. J. Verbal Learn. Verbal Behav. 19 (2), 135-151. doi:10.1016/ S0022-5371(80)90132-2

Green, M. C., and Brock, T. C. (2000). The Role of Transportation in the Persuasiveness of Public Narratives. J. Personal. Soc. Psychol. 79 (5), 701-721. doi:10.1037/0022-3514.79.5.701

Green, M. C., and Donahue, J. K. (2018). The Effects of False Information in News Stories," in Misinformation and Mass Audiences (Austin: University of Texas Press), 109-123.

Hayes, A. (2013). Introduction to Mediation, Moderation, and Conditional Process Analysis: A Regression-Based Approach. New York: The Guilford Press.

Hinyard, L. J., and Kreuter, M. W. (2007). Using Narrative Communication as a Tool for Health Behavior Change: A Conceptual, Theoretical, and Empirical Overview. Health Educ. Behav. 34 (5), 777-792. doi:10.1177/ 1090198106291963
Hoeken, H., Kolthoff, M., and Sanders, J. (2016). Story Perspective and Character Similarity as Drivers of Identification and Narrative Persuasion. Hum. Commun. Res. 42 (2), 292-311. doi:10.1111/hcre.12076

Huskey, R., Mangus, J. M., Turner, B. O., and Weber, R. (2017). The Persuasion Network Is Modulated by Drug-Use Risk and Predicts Anti-drug Message Effectiveness. Soc. Cogn. Affective Neurosci. 12 (12), 1902-1915. doi:10.1093/ scan/nsx126

Imhof, M. A., Schmälzle, R., Renner, B., and Schupp, H. T. (2017). How Real-Life Health Messages Engage Our Brains: Shared Processing of Effective Antialcohol Videos. Soc. Cogn. Affective Neurosci. 12 (7), 1188-1196. doi:10.1093/ scan/nsx044

Koriat, A. (2008). "Metacognition and Consciousness," in The Cambridge Handbook of Consciousness (Cambridge University Press), 289-325.

Lee, E.-J., and Shin, S. Y. (2019). Mediated Misinformation: Questions Answered, More Questions to Ask. Am. Behav. Scientist, 65. 259-276. American Behavioral Scientist. doi:10.1177/0002764219869403

McEwan, B., Carpenter, C. J., and Westerman, D. (2018). On Replication in Communication Science. Commun. Stud. 69 (3), 235-241. doi:10.1080/ 10510974.2018.1464938

McQueen, A., Kreuter, M. W., Kalesan, B., and Alcaraz, K. I. (2011) Understanding Narrative Effects: The Impact of Breast Cancer Survivor Stories on Message Processing, Attitudes, and Beliefs Among African American Women. Health Psychol. 30 (6), 674-682. doi:10.1037/a0025395

Moyer-Gusé, E., and Dale, K. (2017). "Narrative Persuasion Theories," in The International Encyclopedia of Media Effects. Editors P. Rössler, C. A. Hoffner, and L. van Zoonen (John Wiley \& Sons), 1-11. doi:10.1002/ 9781118783764.wbieme0082

Moyer-Gusé, E. (2015). Extending the Examination of Audience Involvement with Media Personae: Response to Brown. Commun. Theor. 25 (3), 284-289. doi:10.1111/comt.12071

Moyer-Gusé, E., and Nabi, R. (2010). Explaining the Effects of Narrative in an Entertainment Television Program: Overcoming Resistance to Persuasion. Hum. Commun. Res. 36 (1), 26-52. doi:10.1111/j.1468-2958.2009.01367.x

Moyer-Gusé, E. (2008). Toward a Theory of Entertainment Persuasion: Explaining the Persuasive Effects of Entertainment-Education Messages. Commun. Theor. 18 (3), 407-425. doi:10.1111/j.1468-2885.2008.00328.x

Murphy, S. T., Frank, L. B., Chatterjee, J. S., and Baezconde-Garbanati, L. (2013). Narrative versus Nonnarrative: The Role of Identification, Transportation, and Emotion in Reducing Health Disparities. J. Commun. 63 (1), 116-137. doi:10.1111/jcom.12007

Niederdeppe, J., Shapiro, M. A., and Porticella, N. (2011). Attributions of Responsibility for Obesity: Narrative Communication Reduces Reactive Counterarguing Among Liberals. Hum. Commun. Res. 37 (3), 295-323. doi:10.1111/j.1468-2958.2011.01409.x

Oliver, M. B., Dillard, J. P., Bae, K., and Tamul, D. J. (2012). The Effect of Narrative News Format on Empathy for Stigmatized Groups. Journalism Mass Commun. Q. 89 (2), 205-224. doi:10.1177/1077699012439020

Oschatz, C., and Marker, C. (2020). Long-term Persuasive Effects in Narrative Communication Research: A Meta-Analysis. J. Commun. 70 (4), 473-496. doi:10.1093/joc/jqaa017

Petty, R. E., and Briñol, P. (2008). Psychological Processes Underlying Persuasion. Diogenes 55 (1), 52-67. doi:10.1177/0392192107087917

Petty, R. E., Briñol, P., Tormala, Z., and Wegener, D. (2007). "The Role of Metacognition in Social Judgments," in Social Psychology: Handbook of Basic Principles (New York: Guilford Press), 254-284.

Petty, R. E., and Cacioppo, J. T. (1986). "The Elaboration Likelihood Model of Persuasion," in Advances in Experimental Social Psychology. 1st ed. (Academic Press), 19, 123-205. doi:10.1016/s0065-2601(08)60214-2

Schwartz, B., and Metcalfe, J. (1994). Methodological Problems and Pitfalls in the Study of Human. MIT Press, 137-156. doi:10.7551/mitpress/4561.003.0007

Schwarz, N. (2010a). "Feelings-as-information Theory," in Handbook of Theories of Social Psychology (Thousand Oaks: Sage Publishing).

Schwarz, N. (2010b). "Meaning in Context: Metacognitive Experiences," in The Mind in Context (New York: Guilford Press), 105-125.

Schwarz, N. (2015). "Metacognition," in APA Handbook of Personality and Social Psychology: Attitudes and Social Cognition (Washington, D.C.: APA), Vol. 1, 203-229. doi:10.1037/14341-006 
Shen, F., Sheer, V. C., and Li, R. (2015). Impact of Narratives on Persuasion in Health Communication: A Meta-Analysis. J. Advertising 44 (2), 105-113. doi:10.1080/00913367.2015.1018467

Shulman, H. C., and Sweitzer, M. D. (2018). Advancing Framing Theory: Designing an Equivalency Frame to Improve Political Information Processing. Hum. Commun. Res. 44 (2), 155-175. doi:10.1093/hcr/hqx006

Slater, M. D., and Gleason, L. S. (2012). Contributing to Theory and Knowledge in Quantitative Communication Science. Commun. Methods Measures 6, 215-236. doi:10.1080/19312458.2012.732626

Slater, M. D., and Rouner, D. (2002). Entertainment?Education and Elaboration Likelihood: Understanding the Processing of Narrative Persuasion. Commun. Theor. 12 (2), 173-191. doi:10.1111/j.1468-2885.2002.tb00265.x

Song, H., and Schwarz, N. (2009). If It's Difficult to Pronounce, it Must Be Risky. Psychol. Sci. 20 (2), 135-138. doi:10.1111/j.1467-9280.2009.02267.x

Sun, Y., Lee, T. K., and Qian, S. (2019). Beyond Personal Responsibility: Examining the Effects of Narrative Engagement on Communicative and Civic Actions. J. Health Commun. 24 (6), 603-614. doi:10.1080/10810730.2019.1643954

Tal-Or, N., and Cohen, J. (2010). Understanding Audience Involvement: Conceptualizing and Manipulating Identification and Transportation. Poetics 38 (4), 402-418. doi:10.1016/j.poetic.2010.05.004

Tukachinsky, R. (2014). Experimental Manipulation of Psychological Involvement with media. Commun. Methods Measures 8 (1), 1-33. doi:10.1080/ 19312458.2013.873777

Vaughn, L. A., Childs, K. E., Maschinski, C., Paul Niño, N., and Ellsworth, R. (2010). Regulatory Fit, Processing Fluency, and Narrative Persuasion. Social Personal. Psychol. Compass 4 (12), 1181-1192. doi:10.1111/j.1751-9004.2010.00325.x

Walter, N., Bilandzic, H., Schwarz, N., and Brooks, J. J. (2020). Metacognitive Approach to Narrative Persuasion: the Desirable and Undesirable Consequences of Narrative Disfluency. Media Psychol. Media Psychology, 1-27. doi:10.1080/15213269.2020.1789477
Wang, W., and Shen, F. (2019). The Effects of Health Narratives: Examining the Moderating Role of Persuasive Intent. Health Marketing Q. 36 (2), 120-135. doi:10.1080/07359683.2019.1575061

Wirtz, J. G., and Kulpavaropas, S. (2014). The Effects of Narrative and Message Framing on Engagement and Eating Intention Among a Sample of Adult Hispanics. J. Nutr. Edu. Behav. 46 (5), 396-400. doi:10.1016/ j.jneb.2013.12.005

Yeshurun, Y., Swanson, S., Simony, E., Chen, J., Lazaridi, C., Honey, C. J., et al. (2017). Same Story, Different Story. Psychol. Sci. 28 (3), 307-319. doi:10.1177/ 0956797616682029

Zwaan, R. A. (1994). Effect of Genre Expectations on Text Comprehension. J. Exp. Psychol. Learn. Mem. Cogn. 20 (4), 920-933. doi:10.1037/0278-7393.20.4.920

Conflict of Interest: The authors declare that the research was conducted in the absence of any commercial or financial relationships that could be construed as a potential conflict of interest.

Publisher's Note: All claims expressed in this article are solely those of the authors and do not necessarily represent those of their affiliated organizations, or those of the publisher, the editors and the reviewers. Any product that may be evaluated in this article, or claim that may be made by its manufacturer, is not guaranteed or endorsed by the publisher.

Copyright (c) 2021 Bullock, Shulman and Huskey. This is an open-access article distributed under the terms of the Creative Commons Attribution License (CC BY). The use, distribution or reproduction in other forums is permitted, provided the original author(s) and the copyright owner(s) are credited and that the original publication in this journal is cited, in accordance with accepted academic practice. No use, distribution or reproduction is permitted which does not comply with these terms. 